\title{
Miniaturized negative permeability materials
}

Kamil Boratay Alici, Filiberto Bilotti, Lucio Vegni, and Ekmel Ozbay

Citation: Appl. Phys. Lett. 91, 071121 (2007); doi: 10.1063/1.2772672

View online: http://dx.doi.org/10.1063/1.2772672

View Table of Contents: http://aip.scitation.org/toc/apl/91/7

Published by the American Institute of Physics

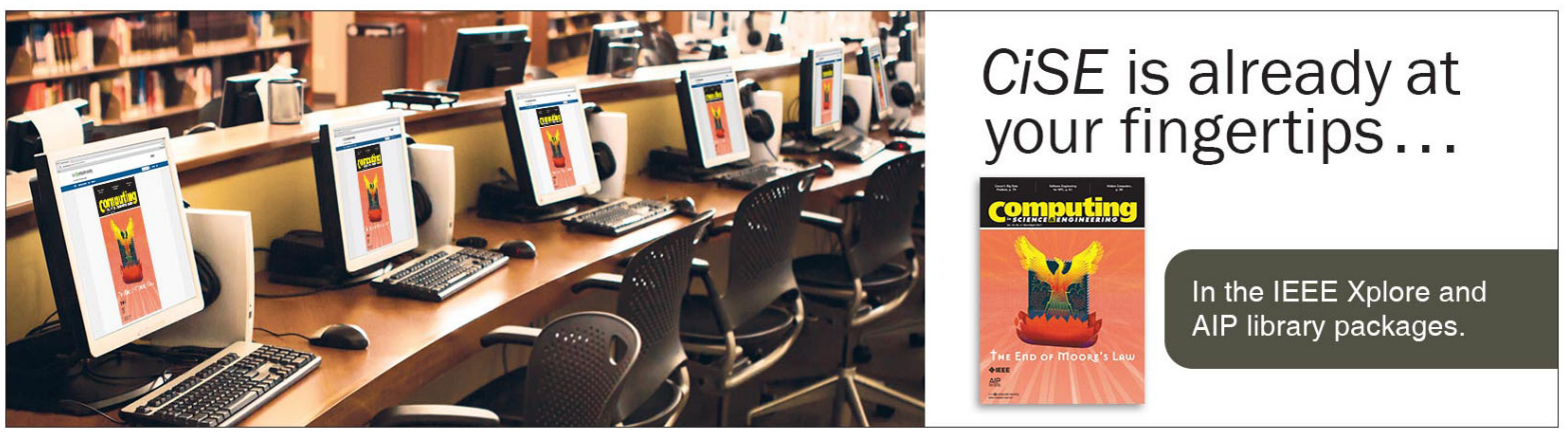




\title{
Miniaturized negative permeability materials
}

\author{
Kamil Boratay Alicia) \\ Nanotechnology Research Center, Bilkent University, 06800 Ankara, Turkey and Department of Physics, \\ Bilkent University, 06800 Ankara, Turkey \\ Filiberto Bilotti and Lucio Vegni \\ Department of Applied Electronics, University of Roma Tre Via della Vasca Navale, 84-00146, Rome, Italy \\ Ekmel Ozbay \\ Nanotechnology Research Center, Bilkent University, 06800 Ankara, Turkey; Department of Physics, Bilkent \\ University, 06800 Ankara, Turkey; and Department of Electrical and Electronics Engineering, Bilkent \\ University, 06800 Ankara, Turkey
}

(Received 30 May 2007; accepted 26 July 2007; published online 17 August 2007)

Experimental and numerical studies of $\mu$-negative (MNG) materials such as multisplit ring resonators (MSRRs) and spiral resonators (SRs) are presented. The resonance frequency of the structures is determined by the transmission measurements and minimum electrical size of $\lambda_{0} / 17$ for the MSRRs and of $\lambda_{0} / 82$ for the SRs observed. These MNG materials can be easily produced by the well developed printed circuit board and optical lithography techniques. They are promising elements for the development of high resolution metamaterial lenses and electrically small antennas. (C) 2007 American Institute of Physics. [DOI: 10.1063/1.2772672]

Electromagnetic metamaterials possess unusual properties, such as the reversal of both Cherenkov radiation and Doppler shift, as well as a negative index of refraction, ${ }^{1}$ which enables subwavelength imaging. ${ }^{2}$ Experimental demonstration of negative refraction is conducted by using a composite medium. ${ }^{3}$ The elements of the medium are thin wires and split ring resonators. The periodic arrangement of the thin wires provides a negative effective permittivity at the frequencies lower than the plasma frequency of the system. ${ }^{4}$ The split ring resonator is an example of magnetic microstructures that are designed to generate a negative effective permeability. ${ }^{5}$ The study of $\mu$-negative (MNG) structures is of considerable interest due to the various imaging and antenna applications.

In magnetic resonance imaging, the introduction of MNG materials improved image quality by guiding the radio frequency flux from an object to a receiver. ${ }^{6}$ The subwavelength image of the second-harmonic field of a source can be obtained by using a metamaterial slab with a quadratic nonlinear response. ${ }^{7}$ Moreover, by using a one-dimensional lefthanded metamaterial, subwavelength imaging at microwave frequencies is demonstrated experimentally. ${ }^{8}$ Loading antennas with MNG materials can improve their performance, especially in terms of achieving smaller sizes. MNG material loaded monopole antenna, ${ }^{9}$ patch antenna, ${ }^{10-12}$ and waveguides $^{13,14}$ are studied in the literature. In all of these applications, the rather small electrical size of the structures is favorable.

The miniaturization of MNG materials is realized by changing the geometry of the structures. The most common MNG material is the split ring resonator. There are two flat disks, each of which are made discontinuous with a split, and which are separated from each other by a gap. ${ }^{5}$ Instead of flat disks, long cylindrical sheets can also be used. ${ }^{5,15}$ In order to increase the distributed capacitance, cylindrical sheets are wound as coils, in which the structure is called a Swiss roll. $^{5,6}$ The flat version of the Swiss roll is called the spiral

\footnotetext{
${ }^{a)}$ Electronic mail: bora@fen.bilkent.edu.tr
}

resonator, ${ }^{10,16}$ which is commonly used as a lumped inductor in microwave engineering. ${ }^{17}$ In addition to changing the geometry of the structures, one can introduce lumped elements to the existing structures. A capacitance loaded loop, ${ }^{18}$ ring, ${ }^{19}$ double sided spiral, ${ }^{20}$ and split ring resonators ${ }^{21}$ can be found in the literature.

However, in most of the above examples, fabrication is a difficult and tedious procedure. The arrays of Swiss rolls, long cylindrical sheets, and capacitively loaded elements cannot be produced easily. Moreover, the Swiss rolls and long cylindrical sheet structures are strongly anisotropic, and due to the purely electrical effects at the axis of the cylinders, they respond as an effective metal when the incident wave polarization is at the axis of the cylinders. On the other hand, the structures, which are considerably thin at the axis of cylinders, can be packed into arrays in three dimensions. By this technique an isotropic medium can be obtained in principle. ${ }^{5}$

In the present work, we study electrically small MNG materials that can be produced relatively easily in the domain of well developed printed circuit board manufacturing techniques and optical lithography processes. The structures are multisplit ring resonators (MSRRs) and a version of spiral resonators (SRs). The MSRR consists of concentric split rings that are designed in order to increase the distributed capacitance between the strips [Fig. 1(a)]. The geometrical parameters of the structure are as follows: the side length of the outer ring $l=8 \mathrm{~mm}$, the strip width $w=100 \mu \mathrm{m}$, the sepa-
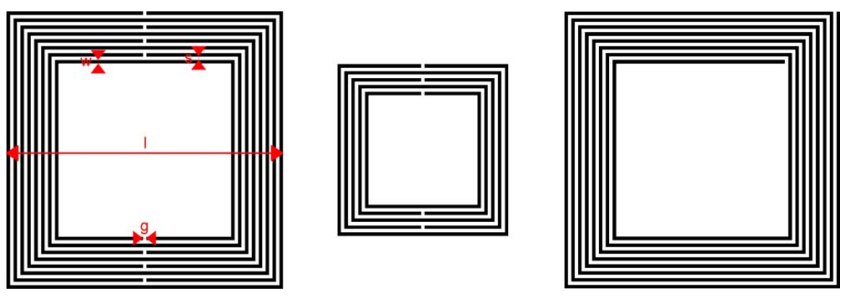

FIG. 1. (Color online) Geometry of examples of MNG materials: (a) Multisplit ring resonator with side length $l=8 \mathrm{~mm}$ and number of rings $N=8$; (b) multisplit ring resonator with $l=5, N=5$; (c) spiral resonator with $l=8 \mathrm{~mm}$ and number of turns $N=8$. 
TABLE I. Comparison of the MNG materials in the literature in terms of electrical size $(u)$, resonance frequency $\left(f_{0}\right)$, and radius of the minimum sphere $(a)$. The free space wavelength is denoted as $\lambda_{0}$. (Capacitance loaded is abbreviated as C.L.) The exact numbers of rings (turns) given in the last three columns are $N=12$ for the MSRR structures and $N=20$ for the SR structure.

\begin{tabular}{|c|c|c|c|c|c|c|c|c|c|}
\hline & $\begin{array}{c}\text { Swiss } \\
\text { roll }\end{array}$ & $\begin{array}{l}\text { Cylindrical } \\
\text { SRR }\end{array}$ & $\begin{array}{l}\text { C.L. } \\
\text { loop }\end{array}$ & $\begin{array}{l}\text { C. L. } \\
\text { ring }\end{array}$ & $\begin{array}{l}\text { C.L. double } \\
\text { sided spiral }\end{array}$ & $\begin{array}{l}\text { C.L. } \\
\text { SRR }\end{array}$ & $\begin{array}{c}\text { MSRR } \\
(1=8)\end{array}$ & $\begin{array}{c}\text { MSRR } \\
(1=5)\end{array}$ & $\begin{array}{c}\text { SR } \\
(1=8)\end{array}$ \\
\hline$a(\mathrm{~mm})$ & 100.1 & 8.2 & 6.8 & 14.0 & 6.5 & 3.7 & 5.7 & 3.5 & 5.7 \\
\hline$f_{0}(\mathrm{MHz})$ & 22.1 & 1440.0 & 60.0 & 46.2 & 156.4 & 990.0 & 1545.0 & 3105.0 & 323.5 \\
\hline$u\left(\lambda_{0}\right)$ & $1 / 68$ & $1 / 13$ & $1 / 367$ & $1 / 232$ & $1 / 148$ & $1 / 41$ & $1 / 17$ & $1 / 14$ & $1 / 82$ \\
\hline
\end{tabular}

ration between adjacent strips $s=100 \mu \mathrm{m}$, and the split width $g=100 \mu \mathrm{m}$. One other example of the MSRRs is shown in Fig. 1(b), in which all of the parameters are the same, excepting the side length of the outer ring $(l=5 \mathrm{~mm})$. In Fig. 1(c) the SR geometry is shown, in which we also have $s=w=100 \mu \mathrm{m}$ and $l=8 \mathrm{~mm}$. A parameter of considerable interest for the MSRRs (SRs) is $N$ : number of strips (number of turns). We kept the dimensions of the structures fixed while changing the number of strips.

These MNG materials are fabricated on a RT/duroid 5880 substrate with listed relative permittivity $\varepsilon=2.0$ and dissipation factor $\tan \delta=0.0009$ at $10 \mathrm{GHz}$. The thickness of the printed circuit board (PCB) was $254 \mu \mathrm{m}$ and the deposited copper thickness on the board was $18 \mu \mathrm{m}$. The MNG materials were obtained by properly etching the metal deposit of the PCB.

At this point, it is necessary to identify a standard for the determination of the size of the MNG materials. We follow a rather fundamental paper that discusses the theoretical limits of the antennas. ${ }^{22}$ While defining the electrical size of a structure, we consider the minimum sphere that can enclose it. If the radius of the sphere is $a$, then the larger linear dimension of the structure is $2 a$. The electrical size $(2 a)$ is identified in terms of the free space wavelength $\left(\lambda_{0}\right)$ at which the structure operates: $u=2 a / \lambda_{0}$. The calculated minimum radius $(a)$ and electrical size $(u)$ of the miniaturized MNG materials in the literature are shown in Table I.

In the present work, the resonance frequencies of the MNG materials are calculated numerically by using the commercial software CST MICROWAVE STUDIO. This tool is a three-dimensional full-wave solver employing the finite integration technique. ${ }^{23}$ We excite a layer of MNG materials with a plane wave and obtain the transmission amplitudes. The dip of the transmission data gives an estimate of the resonance frequency of the structure. The structure shows the MNG behavior after the resonance frequency.

The resonant response of an single MNG material is measured by using two coaxial probes connected to an HP8510C network analyzer. First, we measure the transmission spectra of the free space, i.e., without the MNG material. We use these data for calibration and then repeat the experiment with the MNG material inserted (Fig. 2). The positive transmission values in Fig. 3 are due to the calibration technique and the lensing effect of the structures. The distance between the receiver and transmitter probes is kept fixed during the measurements.

The experimental and numerical results are shown in Figs. 3 and 4. For the MSRR and SR materials, as we increase the number of rings or number of turns, the resonance frequency shifts toward smaller values. The miniaturization factor for the SRs is higher than the MSRRs. In Fig. 4, we see that increasing the number $N$ above a critical point does not reduce the resonance frequency anymore. The resonance frequency of the MSRR can be significantly reduced up to $4-5$ rings $(N=5)$. From Fig. 4 we conclude that it is not necessary to completely fill the inner part of the SR in order to obtain a good reduction of the resonance frequency. Similar to the case of the MSRR, we see that after some point, increasing the number $N$ does not affect the resonance frequency. The calculated electrical size $(u)$, radius of the minimum sphere $(a)$, and resonance frequency $\left(f_{0}\right)$ for the optimum structures are shown in Table I. The reduction of the resonance frequency is comparable with the examples found in the literature. The MNG materials are relatively easy to fabricate, low profile, and thereby can be packed into arrays in several dimensions. For the two MSRR examples, we see that increasing the number of rings can reduce the electrical size. This principle is also valid for the SRs. Moreover, using a higher permittivity substrate will lead us to the further reduction of the resonance frequency. These results can be explained by the aid of theoretical analysis and modeling of the SRs in literature. ${ }^{24,25}$ Moreover, a detailed analysis and circuit model of our structures is given in Ref. 26.

The incident electromagnetic wave induces current on the resonators. At the resonance frequency, the electric and magnetic energy in the structure increases dramatically. Since the structures are small compared to the wavelength, the results can be explained by a quasistatic approach. We consider the change of the total inductance $(L)$ and capacitance $(C)$ of the structures as the number of rings or turns increases. The significant parameters to determine the $L$ and $C$ are the average length of the strips and their filling ratio. ${ }^{26}$ There are three important results to be explained: the decrease of the resonance frequency, miniaturization factor difference between the MSRR and SR structures, and the saturation behavior.

As $N$ gets larger values the capacitance of the structures increases while the inductance decreases. Since the proportion decrease of the inductance is smaller than the proportion

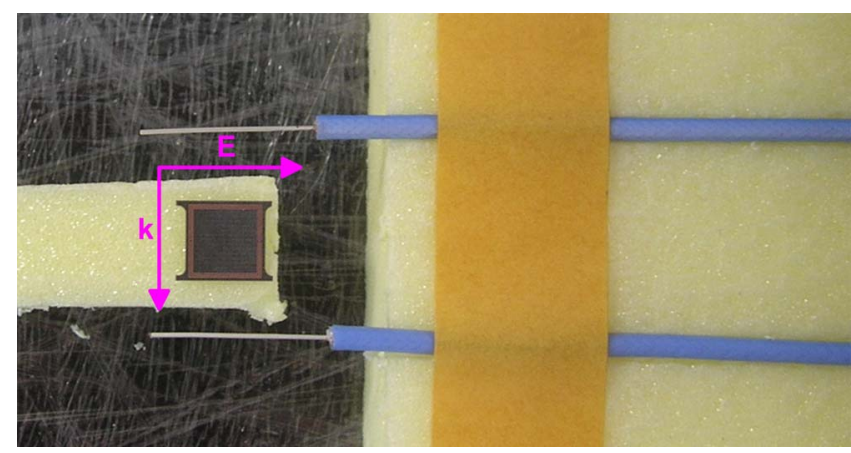

FIG. 2. (Color online) Experiment setup (photo). The polarization of the incident wave is also shown. 

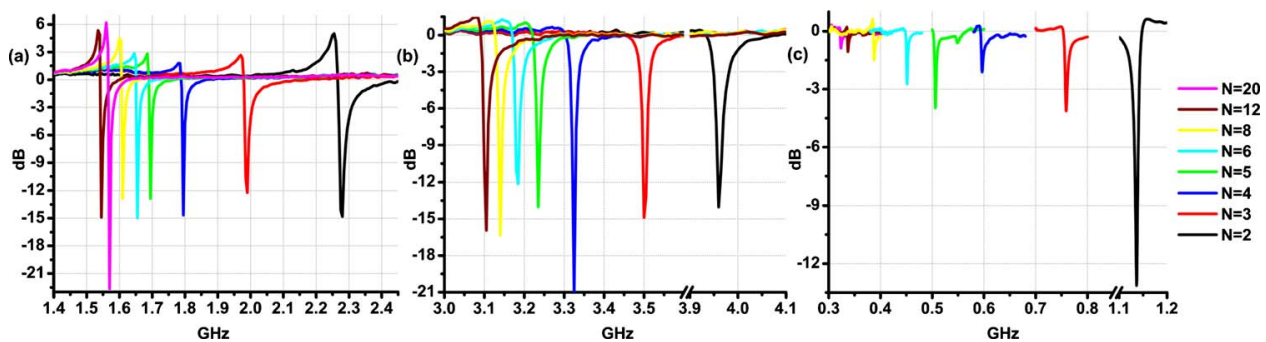

FIG. 3. (Color online) Experimental transmission data as a function of the frequency. (a) Multisplit ring resonators with the side length $l=8 \mathrm{~mm}$. (b) Multisplit ring resonators with the side length $l=5 \mathrm{~mm}$. (c) Spiral resonators with the side length $l=8 \mathrm{~mm}$. increase of the capacitance, we observe a shift of the resonance frequency to lower values. The proportion capacitance difference between the MSRRs and SRs is due to the split capacitance of the MSRRs. The total split capacitance of the MSRRs is significantly smaller than the distributed one. Therefore, as we change the number of rings (turns), the proportion capacitance change of the MSRRs and SRs shows a similar behavior. The miniaturization factor difference of the MSRRs and SRs is related to inductance. ${ }^{26}$ For the MSRRs in addition to the average length of the strips, the filling ratio has an additional decreasing effect on the inductance. Therefore, the proportion decrease of the inductance is higher for the MSRRs that give a smaller miniaturization factor. The saturation of the resonance frequency is due to the saturation of both the inductance and the capacitance of the structures. The average length and the filling ratio increase with a decreasing rate, which yields a saturation behavior. $^{26}$

In summary, we experimentally demonstrated electrically small MNG materials. For the MSRR structures, the electrical size of $\lambda_{0} / 17$ and for the SR structures electrical size of $\lambda_{0} / 82$ is achieved. Further size reduction can be realized by increasing the number $N$ or using a higher permittivity substrate. The MNG materials are relatively easy to fabricate, low profile, and can be packed into arrays. Since the electrical size of the metamaterial element limits the

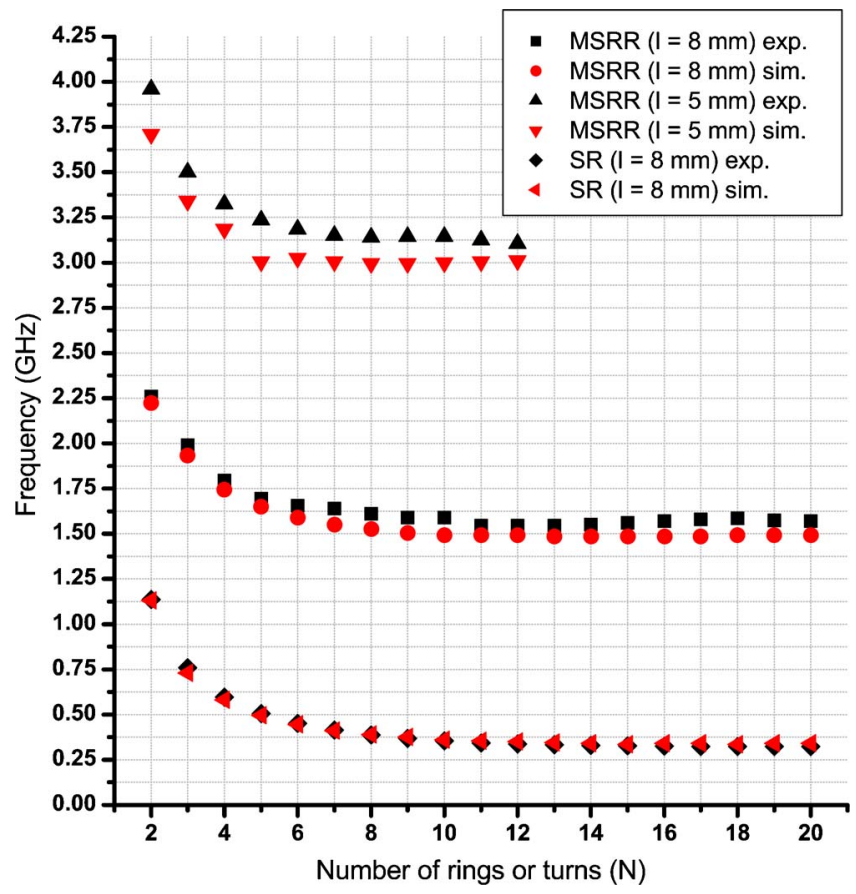

FIG. 4. (Color online) Resonance frequency as a function of the number of rings and turns (experiment and simulation). resolution of the subwavelength imaging, the MNG materials demonstrated here can be the first step toward the metamaterial based ultrahigh resolution imaging systems. Moreover, they establish a solution for the antenna miniaturization problem in wireless systems.

This work is supported by the European Union under the projects EU-NoE-METAMORPHOSE, EU-NoEPHOREMOST, and TUBITAK under Project Nos. 104E090, 105E066, 105A005, and 106A017. One of the authors (E.O.) also acknowledges partial support from the Turkish Academy of Sciences.

${ }^{1}$ V. G. Veselago, Sov. Phys. Usp. 10, 509 (1968).

${ }^{2}$ J. B. Pendry, Phys. Rev. Lett. 85, 3966 (2000).

${ }^{3}$ D. R. Smith, W. J. Padilla, D. C. Vier, S. C. Nemat-Nasser, and S. Schultz, Phys. Rev. Lett. 84, 4184 (2000).

${ }^{4}$ J. B. Pendry, A. J. Holden, W. J. Stewart, and I. Youngs, Phys. Rev. Lett. 76, 4773 (1996).

${ }^{5}$ J. B. Pendry, A. J. Holden, D. J. Robbins, and W. J. Stewart, IEEE Trans. Microwave Theory Tech. 47, 2075 (1999).

${ }^{6}$ M. C. K. Wiltshire, J. B. Pendry, I. R. Young, D. J. Larkman, D. J. Gilderdale, and J. V. Hajnal, Science 291, 849 (2001).

${ }^{7}$ A. A. Zharov, N. A. Zharova, I. V. Shadrivov, and Yu. S. Kivshar, Appl. Phys. Lett. 87, 091104 (2005).

${ }^{8}$ A. N. Lagarkov and V. N. Kissel, Phys. Rev. Lett. 92, 077401 (2004).

${ }^{9}$ K. B. Alici and E. Ozbay, J. Appl. Phys. 101, 083104 (2007).

${ }^{10}$ K. Buell, H. Mosallaei, and K. Sarabandi, IEEE Trans. Microwave Theory Tech. 54, 135 (2006).

${ }^{11}$ A. Alù, F. Bilotti, N. Engheta, and L. Vegni, IEEE Trans. Antennas Propag. 55, 13 (2007).

${ }^{12} \mathrm{P}$. Ikonen, M. Karkkainen, and S. Tretyakov, IEEE AP-S International Symposium, Washington DC, 3-8 July 2005 (IEEE, New York, 2005), Vol. 2a, pp. 606-609.

${ }^{13}$ S. Hrabar, J. Bartolic, and Z. Sipus, IEEE Trans. Antennas Propag. 53, 110 (2005).

${ }^{14}$ R. Marqués, J. Martel, F. Mesa, and F. Medina, Phys. Rev. Lett. 89, 183901 (2002).

${ }^{15}$ M. Shamonin, E. Shamonina, V. Kalinin, and L. Solymar, Microwave Opt. Technol. Lett. 44, 133 (2005).

${ }^{16}$ J. D. Baena, R. Marques, F. Medina, and J. Martel, Phys. Rev. B 69, 014402 (2004)

${ }^{17}$ I. Bahl and P. Bhartia, Microwave Solid State Circuit Design, 2nd ed. (Wiley, New York, 2003), Vol. I, pp. 57-63.

${ }^{18}$ M. C. K. Wiltshire, E. Shamonina, I. R. Young, and L. Solymar, Electron. Lett. 39, 215 (2003).

${ }^{19}$ O. Sydoruk, A. Radkovskaya, O. Zhuromskyy, E. Shamonina, M. Shamonin, C. J. Stevens, G. Faulkner, D. J. Edwards, and L. Solymar, Phys. Rev. B 73, 224406 (2006).

${ }^{20}$ R. R. A. Syms, I. R. Young, and L. Solymar, J. Phys. D 39, 3945 (2006).

${ }^{21}$ K. Aydin and E. Ozbay, J. Appl. Phys. 101, 024911 (2007).

${ }^{22}$ L. J. Chu, J. Appl. Phys. 19, 1163 (1948).

${ }^{23}$ User Manual, Version 5.0, CST GmbH, Darmstadt, Germany, 2005, http:// www.cst.de

${ }^{24}$ S. S. Mohan, M. Hershenson, S. P. Boyd, and T. H. Lee, IEEE J. SolidState Circuits 34, 1419 (1999).

${ }^{25}$ C. R. Simovski and A. A. Sochava, Prog. Electromagn. Res. 43, 239 (2003).

${ }^{26}$ F. Bilotti, A. Toscano, and L. Vegni, IEEE Trans. Antennas Propag. (to be published). 